\title{
Misdiagnosed maxillary sinusitis: the case for further investigation
}

\author{
Valfrido A. Pereira-Filho ${ }^{1}$, Marisa Aparecida Cabrini Gabrielli ${ }^{1}$, Eduardo Hochulli-Vieira ${ }^{1}$, \\ Mario Francisco Real Gabrielli², Leandro Eduardo Klüppel ${ }^{3}$ and Fernando Antonini ${ }^{4 *}$ \\ ${ }^{1}$ Associate Professor, Oral and Maxillofacial Surgery Department, Araraquara Dental School - Sao Paulo State University, Brazil \\ ${ }^{2}$ Professor and Chairman, Oral and Maxillofacial Surgery Department, Araraquara Dental School - Sao Paulo State University, Brazil \\ ${ }^{3}$ Associate Professor, Oral and Maxillofacial Surgery Department, Ponta Grossa State University, Brazil \\ ${ }^{4}$ PhD Student, Oral and Maxillofacial Surgery Department, Pontific Catholic University, Rio Grande do Sul, Brazil
}

Received: December 09, 2014; Accepted: March 02, 2015; Published: April 24, 2015

*Corresponding author: Fernando Antonini, Faculty of Dentistry, PUCRS Avenue Ipiranga, 6681 Parthenon, Porto Alegre - RS 90619-900, Tel: +55 48 99465051; E- mail: drfernandoantonini@gmail.com

\begin{abstract}
Rhinocerebral mucormycosis is a rare life threatening fungal infection, affecting almost exclusively patients with known predisposing conditions such as diabetes mellitus, immunocompromised status, haemochromatosis or major trauma. The purpose of this paper is to report a case of orbit-zygomatic bone mucormycosis involvement in a diabetic patient, previously diagnosed and treated as recurrent maxillary sinusitis. Treatment of the condition required extensive surgical intervention and medical management for a life saving outcome.
\end{abstract}

Keywords: Mucormycosis, Fungal infection, Maxillary sinusitis

\section{Introduction}

Mucormycosis, also known as zygomycosis or phycomycosis, is a rare, devastating, life-threatening opportunistic infection caused by a series of fungi in the Mucorales family that commonly affects people with immune disorders, especially patients with poorly controlled diabetes mellitus, hematologic malignancy, organ transplants, chemotherapy, chronic renal insufficiency, iron or aluminum overload, malnutrition, deferoxamine therapy, and severe burns, although there are cases reported in healthy patients $(1,2)$. They invade tissues with a particular affinity for blood vessels, leading to thrombosis, ischemia and necrosis of the surrounding tissue. These fungi are ubiquitous in bread and fruit molds and may be found in soil, manure, plants, vegetable matter, and air (1).

There is some evidence that they are present in the gastrointestinal and respiratory tracts of healthy individuals. The genera Mucorales that is recognized as human pathogens includes Absidia, Rhizomucor, Mucor and Rhizopus. Morphologically, they are nonseptate, broad hyphae (5 to $50 \mathrm{fxm}$ ), with branches at right angles (3).

Rhizopus is responsible for $60 \%$ of all cases of mucormycosis and $90 \%$ of rhinocerebral mucormycosis, which is the most common and the most fatal syndrome of mucormycosis.
Typically, a patient with rhinocerebral mucormycosis in a poor diabetes control has malaise, retro-orbital headache and fever. Symptoms might progress to include facial or orbital cellulitis, ptosis, proptosis, and ophthalmoplegia. Often patients will have infraorbital anesthesia, corneal anesthesia, and facial nerve palsy. Examination of the oral and nasal cavities may reveal a black eschar on the palate, septum, or turbinate. Frequently decreased visual acuity develops and may progress rapidly to blindness. A deterioration in mental status is an ominous sign, often heralding intracerebral extension of the disease process. These symptoms may develop over a period of several days or may occur as a fulminating process within hours (4-6), Other forms include pulmonary, gastrointestinal, cutaneous, central nervous system, disseminated, and, rarely, miscellaneous (i e, bone, kidney, cardiac, mediastinum, oral) (4). In the majority of cases of rhinocerebral mucormycosis the organism gains access to the patient through the nose. This fungi may, however, enter through any disruption of mucosa or skin (5). The oral involvement of this condition may also occur and has been reported in the literature. As in cutaneous mucormycosis, injured tissue in the oral cavity can be a suitable port of entry (6).

Mucormycosis is known for having a very poor prognosis; however, with aggressive medical and surgical management, survival rates are currently thought to exceed $80 \%(7,8)$. Early detection has been correlated with less tissue destruction and a better overall outcome. Moreover, intravenous antifungal therapy with Amphotericin B, wide surgical debridement of all necrotic tissue, and control of the underlying metabolic disease appear to be the three most important factors in managing mucormycosis and achieving satisfactory outcomes. Early detection has been correlated with less tissue destruction and a better overall outcome $(8,9)$.

The purpose of this paper is to report a case of orbit-zygomatic bone mucormycosis involvement, previously diagnosed and treated as recurrent maxillary sinusitis, in which aggressive surgical treatment associated with high doses of antifungal therapy were required to effectively treat the mentioned pathology. 


\section{Case Report}

A 50 year-old male farm laborer presented to the Oral and Maxillofacial Surgery Department of Araraquara Dental School - São Paulo State University, Brazil, for emergency care of right periorbital swelling. On clinical examination, the patient presented a periorbital edema around the right eye, moderately erythematosus, flutuant and painful on palpation (Figure 1). According to the patient, the mentioned edema was rapidly growing and had started 2 days before. Moreover, the patient presented with ophthalmoplegia as well as paresthesia of the right infra-orbital nerve. The patient further reported that the swelling present was recurrent and had been previously diagnosed by two ENT's as maxillary sinusitis, who prescribed oral antibiotics only.

Fever and tachycardia were remarkable. Mental-status examination revealed normal results. No nasopharyngeal, palatal, oropharyngeal, or aural lesions were noted. The initial presentation mentioned was consistent with preseptal orbital cellulitis on the right side. Medical history and admission laboratory values revealed uncontrolled diabetes $(241 \mathrm{mg} / \mathrm{dl})$, low hemoglobin rate $(10.7 \mathrm{~g} / \mathrm{dL})$ and white blood cells count of 7600 cells.

Axial and coronal CT scans revealed edema anterior to orbital globe, consistent with preseptal cellulitis, and opacification and mucosal thickening of maxillary sinus on the right side, consistent with maxillary sinusitis (Figure 2). Normal brain parenchyma could be noted. Although the medial wall of the nasal cavity and sinus floor seemed involved in a osteolytic pattern, the nasal cavity and ethmoid sinus were clear and no intra-oral findings were evident. The zygomatic bone showed a osteolytic pattern as well, but some areas of bone sclerosis (Figure 3).

The patient was hospitalized immediately and a subcutaneous insulin shot was promptly administred to the patient. All other medical condition were fully assessed and proved unremarkable hence not in need for medical treatment. Twenty-four hours after admission underwent general anesthesia and a right CaldwellLuc approach was performed for maxillary sinus drainage and debridement. Histologic analysis of the excised specimen from the right sinus described hemorrhagic necrotic tissue

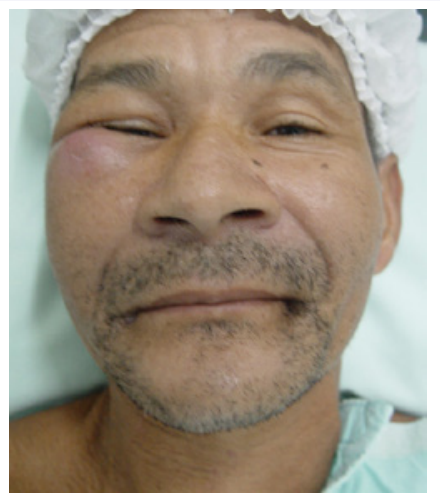

Figure 1: Initial exam shows periorbital edema around the right eye.

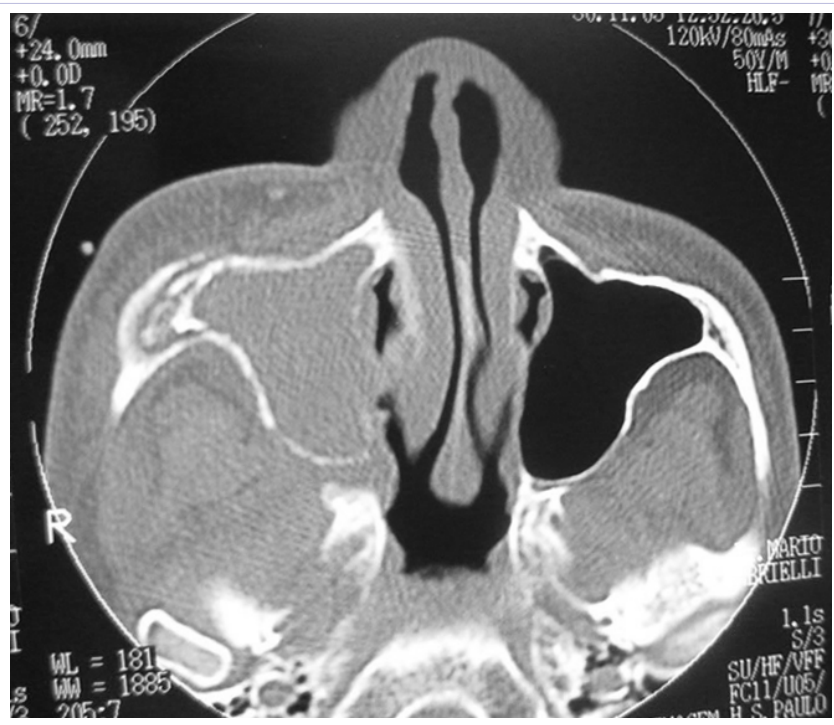

Figure 2: Axial CT scan showing right maxillary sinusitis and preseptal cellulitis.

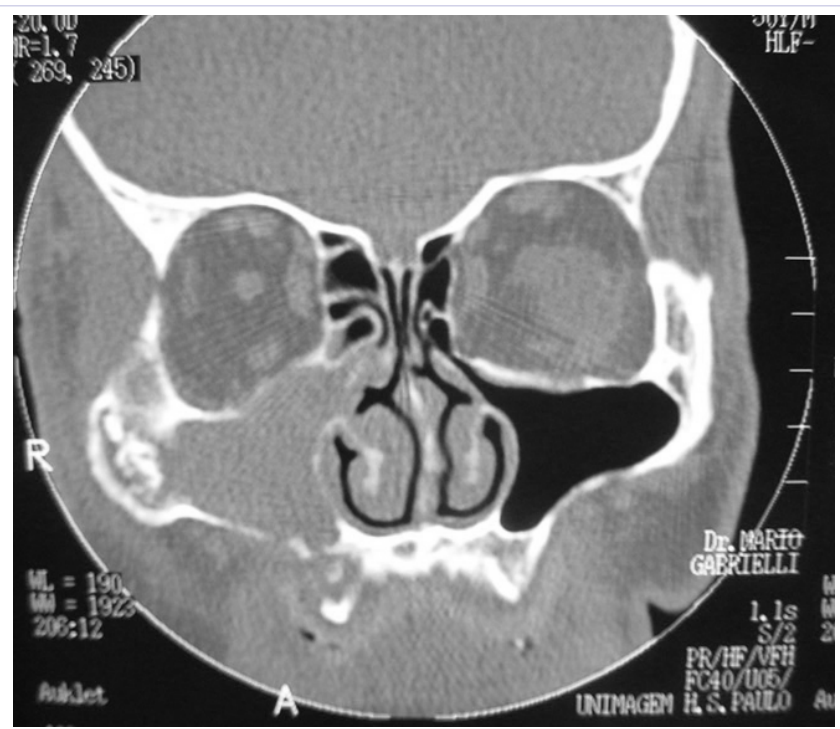

Figure 3: Coronal CT scan showing involvement of the right zygomatic bone in the lesion.

containing broad, scarcely septate fungal hyphae consistent with mucormycosis. Based on the mentioned diagnosis, intravenous amphotericin B therapy (50 mg/day) was initiated and a more radical surgery for zygomatic bone resection was soon performed. Under general anesthesia, a major portion of the zygomatic bone was excised via a lower eyelid and intraoral approaches (Figure $4 \mathrm{~A}$ and $4 \mathrm{~B}$ ). Healthy bone margins were detected and hence considered the limit of the resection, and periorbital soft tissue was sutured around the periorbital rim.

The antifungal therapy lasted 22 days. The initial dose was $50 \mathrm{mg}$ once a day although it had to be elevated to $150 \mathrm{mg}$ daily in order to maintain its efficacy. Immediate post-operative period during hospital stay and after hospital discharge were uneventful. Medical exams show normal and stabilized renal 

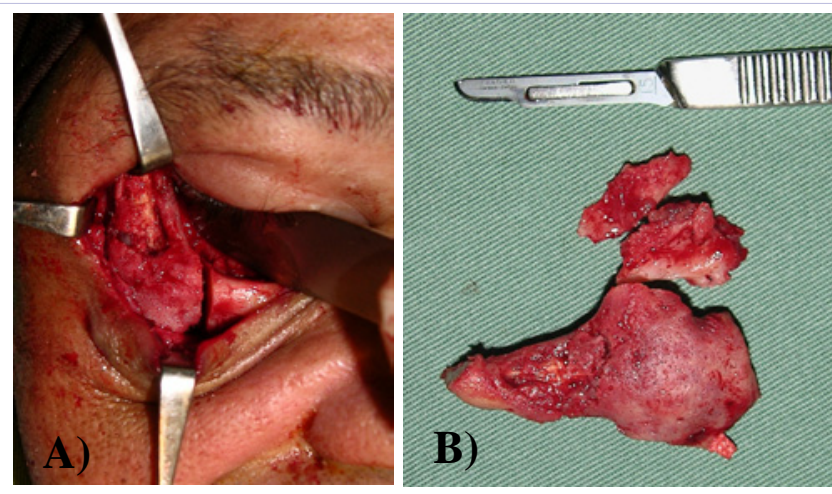

Figure 4: Excision of the zygomatic bone via lower eyelid approach and the excised surgical specimen.

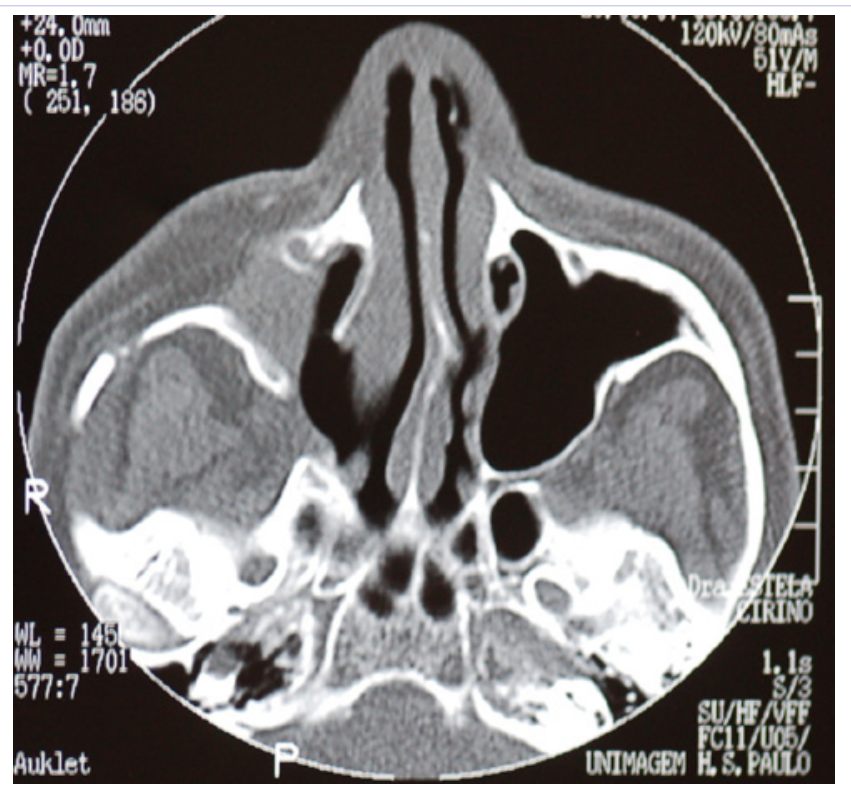

Figure 5: Eighteen months post-operative axial CT scan showing normal bone healing and no relapse of the lesion.

function and the patient has no complaints related to the right eye and its functions. Post-operative CT scans show no relapse of the pathology (Figure 5 and 6). A residual small facial asymmetry is present, although the patient feel comfortable with the deformity eighteen months after zygomatic bone excision (Figure 7 and 8).

\section{Comments}

Rhinocerebral involvement of Mucorales fungi has been extensively reported $(9,11-14)$, and less commonly the oral involvement has also been known. Bonifaz, Macias and ParedesFarrera (6) reported that the oral involvement of mucormycosis can occur at different levels. Although, the involvement of maxillary sinus in this condition has been reported relatively frequently, no case of isolated zygomatic bone and maxillary sinus has been reported without compromising nasal and intracranial structures.

The infection usually occurs by inhalation through the nose or mouth, but skin lacerations can also became a port of entry of the fungi. Although it cannot be fully confirmed, the major suspicion of contamination is nasal inhalation since patient's work environment could potentiate such infection. Nearly all patients receiving the diagnosis of mucormycosis have some underlying disease predisposing them to this opportunistic infection. Between $70 \%$ and $80 \%$ of these patients have varying degree of uncontrolled diabetes mellitus $(8,13)$, as shown in the patient reported in this paper, although the presence of ketoacidosis in a poorly controlled case of diabetes appears to be more important than the mere diagnosis of diabetes (14). Diabetes mellitus alters the normal immunologic response to fungal infection in several ways. Studies in mice and rabbits have shown that acidotic alloxan-induced diabetes mellitus increases susceptibility to fungal infection, while nonacidotic alloxaninduced diabetes mellitus does not (15). Ketotic acidosis alters the polymorphonuclear leukocyte response to mucormycosis. Granulocyte phagocytic ability is reduced in the acidotic state but returns to normal with correction of the acidosis $(16,17)$, as seen also in patients with chronic renal disease. In addition, the ability of serum from diabetic patients to inhibit Rhizopus in vitro is less than that of serum from normal patients (18). However, Waldorf (19) have reported that hyperglycemia or acidosis per se is not sufficient to induce fungal replication within the macrophage and normal human serum can inhibit the growth of Rhizopus, as supported by Chinn and Diamons (20). In contrast, Gale and Welch (18) pointed that serum obtained from patients with diabetic ketoacidosis is not inhibitory and may actually enhance fungal growth. Pillsbury and Fischer state that mucormycosis must be suspected in all immunodepressed or diabetic patients who develop acute sinusitis or facial cellulitis, independent of their metabolic status (9). An important paper published by Martines et al. reported rhinocrerebral involvement by the diagnosis of a uncommon intracranial complication

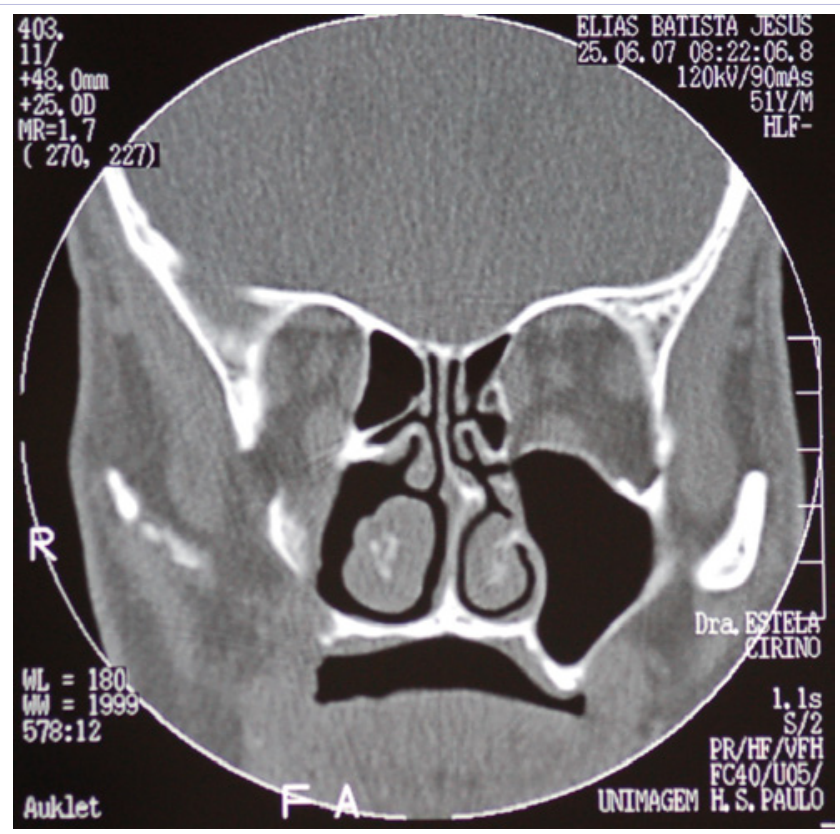

Figure 6: Eighteen months post-operative axial CT scan showing normal bone healing and no relapse of the lesion. 


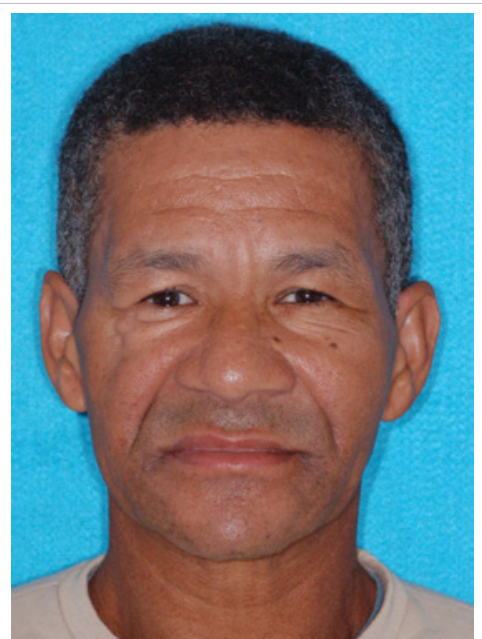

Figure 7: Eighteen months post-operative coronal CT scan showing normal bone healing and no relapse of the lesion.

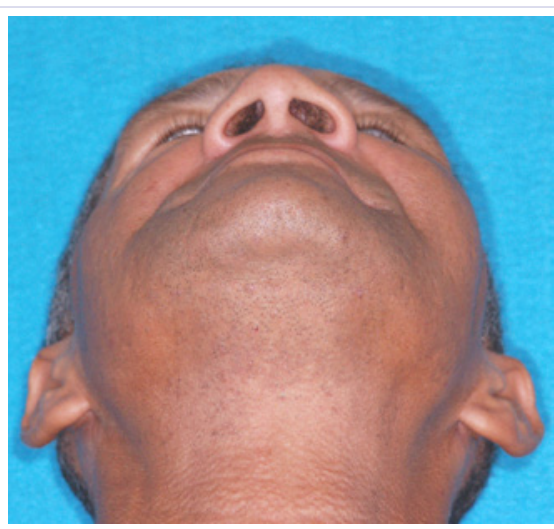

Figure 8: Eighteen months post-operative follow-up showing acceptable esthetics with mild facial asymmetry.

following acute odontogenic sinusitis, emphasizing the role of immunocompromission in the development of such rare and dangerous condition.

Maxillary sinusitis is a multifactor disease with pathophysiological mechanisms that remain unclear and a high prevalence worldwide. The etiology of these diseases is still controversial, although there is a consensus among physicians that the etiopathogenesis is multifactorial and the virulence of the organisms involved is influenced by exogenous and endogenous factors. Infections induce inflammation of the sinus mucosa that can extend to the maxillofacial bones causing osteitis, further worsening symptoms and contributing to the evolution of the disease $(12,13)$. Fungal rhinosinusitis is a form of chronic rhinosinusitis, which is an inflammatory disorder of the nose and paranasal sinuses. It is clinically defined as persistence of symptoms of nasal blockage or discharge for at least 12 weeks combined with endoscopic abnormalities (polyps, mucopurulent discharge, mucosal swelling) or an abnormal sinus on computed tomography (CT) scan findings; it may be primary or secondary fungal infection. Other symptoms may include facial pain or a reduced sense of smell (18). While allergy and bacterial infection play a role in the etiology of this clinical condition, it is best considered as a multifactorial chronic inflammatory disorder. In this case reported, the patient was admitted to the hospital presenting uncontrolled type-II diabetes, fever, tachycardia and showing some clinical signs of preseptal orbital cellulitis which developed over a period of 3 days. Acute maxillary sinusitis could present similar signs and symptoms and hence the diagnosis of the disease presented can become a challenge for many surgeons.

Early evidence of the presence of the disease could also be found on CT scans of the superior and middle thirds of the face. The right maxillary sinus was fully invaded and zygomatic bone involvement could be noted as the fungus began to invade adjacent bone. Direct penetration and growth through the blood vessel wall account for the propensity for thrombosis and tissue necrosis, as occurred in the patient reported. Moreover, during radiological assessment, CT or MRI scans may be necessary to delineate the extent of disease and it is particularly important to identify the presence of intracranial disease. Clinical examination and CT scan were unremarkable for intra-cranial extension of the disease. Laboratory studies are nonspecific. The differential diagnosis should include the following entitles: acute bacterial sinusitis with orbital involvement, orbital cellulitis, aspergillosis, candidiasis, cavernous sinus thrombosis, tuberculosis, midline facial granuloma, antral neoplasia, cellulitis and odontogenic abscesses (9,21). Definitive diagnosis requires histologic examination of involved tissue and visualization of broad, nonseptate hyphae with right angle branching. In this case, a tissue specimen was obtained surgically from the maxillary sinus and presented the mentioned histological characteristics.

After definitive diagnosis, amphotericin B and early aggressive surgical debridement are the treatments of choice. The underlying medical problems also must be aggressively assessed. Volume repletion and acidosis correction in the diabetic patient enhance survival rates. Blitzer et al. (8) analyzed survival factors in patients with mucormycosis. They found an overall survival rate of $50 \%$ in the 179 cases reviewed. Survival rates depend on early identification and treatment. The three most important factors leading to the improved survival appeared to be administration of antifungal therapy, early aggressive surgical debridement, and correction he patient's underlying condition. In diabetics the overall survival rate was $60 \%$, and in patients with other systemic disorders, such as leukemia or renal failure, the survival was $20 \%$.

Antifungal treatment is based on systemic high-dose amphotericin B. The use of amphotericin B improved survival in diabetics to $79 \%$, as compared with $37 \%$ in patients not receiving the drug (8). Because amphotericin B has some serious side effects, in particular renal toxicity, careful monitoring of serum urea nitrogen and creatinine and of creatinine clearance should be carried out during amphotericin B therapy.

Extensive surgical debridement improved survival to $78 \%$. as compared with $57.5 \%$ in patients without aggressive surgical debridement (8). Pillsbury and Fischer (9) found an 85\% long- 
term survival rate in patients treated with early, aggressive surgical debridement and amphotericin B. Blitzer et al. (9) found a survival rate of $89 \%$ in diabetics treated with amphotericin B and "radical surgery."

Tissue necrosis usually develops early in the course of mucormycosis. The early debridement or resection of all necrotic tissue may require radical approaches, including total maxillectomy and orbital exenteration in the rhinocerebral form and partial or total mandibulectomy in mandibular form of mucormcosis. These treatments support the surgical procedure performed in the presented case. Furthermore, the fungus thrives in necrotic tissue, and the vascular thrombosis prevents amphotericin B from reaching the fungus in these tissues. Thus, surgical debridement or resection removes a focus of infection that cannot be treated adequately by systemic medication alone. Even with successful treatment, Mucorales can become dormant and reappear during future courses of chemotherapy and neutropenia (19). More recently, hyperbaric oxygen therapy has been introduced in the treatment of rhinocerebral and atypical forms of mucormycosis. Ferguson et al. (22) have reported increased survival rates with the use of hyperbaric oxygen therapy as an adjunct to the three treatment modalities mentioned above.

Because the initial signs and symptoms of the disease often involve the oral, facial, and cranial structures, it is critical that dentists, dental specialists, physicians, ENT specialists, pathologists, infection doctors and neurologists be aware of this infection, especially in the diabetic or immunocompromised patient. Decisions related to the treatment planning of isolated orbit-zygomatic mucormycosis may be difficult for clinicians because there are no controlled studies that identify when conservative or more aggressive treatment strategies should be used. Thus, clinicians must rely on case reports, personal experience, and clinical judgment.

\section{References}

1. Hauman CItJ, Raubenheimer EJ. Orofacial mucormycosis. Oral Surg Oral Med Oral Pathol 1989; 68:624-7.

2. Pagano L, Offidani M, Fianchi L, et al. Mucormycosis in hematologic patients. Haematologica. 2004; 89(2):207-14.

3. Tugsel Z, Sezer B, Akalin T. Facial swelling and palatal ulceration in a diabetic patient. Oral Surg Oral Med Oral Pathol Oral Radiol Endod. 2004; 98(6):630-6.

4. McDermott NE, Barrett J, Hipp J, Merino MJ, Richard Lee CC, Waterman $P$, et al. Successful treatment of periodontal mucormycosis: report of a case and literature review. Oral Surg Oral Med Oral Pathol Oral Radiol Endod. 2010; 109(3):e64-9. doi: 10.1016/j.tripleo.2009.11.012.

5. Brown OE, Finn R. Mucormycosis of the mandible. J Oral Maxillofac Surg 1986; 44(2):132-6.
6. Bonifaz A, Macias B, Paredes-Farrera F, Arias P, Ponce RM, Araiza J. Palatal zygomycosis: Experience of 21 cases. Oral Dis. 2008; 14(6):569-74. doi: 10.1111/j.1601-0825.2007.01433.x.

7. Pizzo PA. Management of fever in patients with cancer and treatmentinduced neutropenia. N Engl J Med. 1993 ;328(18):1323-32.

8. Blitzer A, Lawson W, Meyers BR, Billet HF. Patient survival factors in paranasal sinus mucormycosis. Laryngoscope. 1980; 90(4):635-48.

9. Pillsbury HC, Fischer ND. Rhinocerebral mucormycosis. Arch Otolaryngol 1977; 103:600-4.

10. Paltauf A. Mycosis mucorina. l'irchows Arch. f. path. Anat. 1885; 102: 543.

11. Finn DG. Mucormycosis of the paranasal sinuses. Ear Nose Throat J. 1988; 67(11):813, 816-8, 821-2.

12. Islam MN, Cohen DM, Celestina LJ, Ojha J, Claudio R, Bhattacharyya IB. Rhinocerebral zygomycosis: An increasingly frequent challenge: Update and favorable outcomes in two cases. Oral Surg Oral Med Oral Pathol Oral Radiol Endod. 2007; 104(5):e28-34.

13. Van Johnson E, Kline LB, Julian BA, Garcia JH. Bilateral cavernous sinus thrombosis due to mucormycosis. Arch Ophthalmol. 1988; 106(8):1089-92.

14. Abedi E, Sismanis A, Choi K, Pastore P. Twenty-five years' experience treating cerebro-rhino-orbital mucormycosis. Laryngoscope. 1984; 94(8):1060-2.

15. Sheldon WH, Bauer H. Activation of quiescent mucormycosis granulomata in rabbits by induction of acute alloxan diabetes. J Exp Med 1958; 108(1):171-8.

16. Bauer H, Flanagan JF, Sheldon WH. Experimental cerebral mucormycosis in rabbits with alloxan diabetes. Yale J Biol Med 1955; 28(1):29-36.

17. Bybee JD, Rogers DE. The phagocytic activity of polymorphonuclear leukocytes obtained from patients with diabetes mellitus. J Lab Clin Med. 1964; 64:1-13.

18. Gale GR, Welch AM. Studies of opportunistic fungi. I. Inhibition of Rhizopus oryzae by human serum. Am J Med Sci. 1961; 241:604-12.

19. Waldorf AR. Host-parasite relationship in opportunistic mycoses. Crit Rev Microbiol. 1986; 13(2):133-72.

20. Chinn RY, Diamond RD. Generation of chemotactic factors by Rhizopus oryzae in the presence and absence of serum: Relationship to hyphal damage mediated by human neutrophils and effects of hyperglycemia and ketoacidosis. Infect Immun. 1982; 38(3):1123-9.

21. Martines F, Salvago P, Ferrara S, Mucia M, Gambino A, Sireci F. Parietal subdural empyema as complication of acute odontogenic sinusitis: a case report. J Med Case Rep. 2014; 8:282. doi: 10.1186/1752-19478-282.

22. Ferguson BJ, Mitchell TG, Moon R, Camporesi EM, Farmer J. Adjunctive hyperbaric oxygen for treatment of rhinocerebral mucormycosis. Rev Infect Dis. 1988; 10(3):551-9. 